\title{
氧化石墨烯吸附水体中酚类有机污染物的分子动力学模拟
}

\author{
赵超锋 ${ }^{1}$, 金佳人 ${ }^{1}$, 霍英忠 ${ }^{1}$, 孙 陆 $^{2}$, 艾玥洁 ${ }^{1}$
}

(1. 华北电力大学 环境科学与工程学院, 资源环境系统优化教育部重点实验室, 北京 $102206 ; 2$. 南开大学现代 光学研究所, 天津 300350)

摘 要: 研究采用分子动力学模拟(Molecular dynamics simulation, MD)的方法, 以苯酚、 $\alpha$-䒺酚和 4-辛基酚为代表, 研究了酚类有机污染物(Phenolic Organic Pollutants, POPs)在氧化石墨烯(Graphene Oxide, GO)上单独和竞争吸附过 程。通过自由能计算得到三种 POPs 在 GO 表面的吸附能分别为: 4-辛基酚 $(41.34 \mathrm{~kJ} / \mathrm{mol})>\alpha$-菜酚 $(33.23 \mathrm{~kJ} / \mathrm{mol})>$ 苯 酚 $(19.31 \mathrm{~kJ} / \mathrm{mol})$ 。吸附过程中的主要作用力为 POPs 的疏水作用, 而分子团簇、范德华相互作用、静电相互作用以 及氢键等在一定程度上增加了 GO 对 POPs 的吸附能力。在混合体系中, POPs 之间存在明显的竞争吸附现象, 吸附 过程包含了直接吸附和形成分子团簇的间接性吸附两个过程。本研究结果为含 POPs 水体的治理以及 GO 材料的设 计和笁选提供了一定的理论依据。

关 键 词: 酚类有机污染物; 氧化石墨烯; 竞争吸附; 分子动力学模拟

中图分类号: X703 文献标识码: A

\section{Adsorption of Phenolic Organic Pollutants on Graphene Oxide: Molecular Dynamics Study}

\author{
ZHAO Chaofeng ${ }^{1}$, JIN Jiaren ${ }^{1}$, HUO Yingzhong ${ }^{1}$, SUN Lu$^{2}$, AI Yuejie ${ }^{1}$
}

(1. MOE Key Laboratory of Resources and Environmental Systems Optimization, College of Environmental Science and Engineering, North China Electric Power University, Beijing 102206, China; 2. Institute of Modern Optics, Nankai University, Tianjin 300350, China)

\begin{abstract}
In this work, molecular dynamics (MD) simulations were applied to address the major concerns about the independent and competitive adsorption processes of phenolic organic pollutants (POPs) on the graphene oxide (GO) in aqueous solution. Phenol, $\alpha$-naphthol and 4-octyl-phenol were adopted as representatives of POPs and their adsorption energies were calculated, which followed an order of 4-octyl-phenol $(41.34 \mathrm{~kJ} / \mathrm{mol})>\alpha$-naphthol $(33.23 \mathrm{~kJ} / \mathrm{mol})>$ phenol $(19.31 \mathrm{~kJ} / \mathrm{mol})$. The simulation results showed that hydrophobic properties of POPs were recognized as the driving force for their adsorption behaviors. Moreover, van der Waals interaction, electrostatic interaction, as well as hydrogen bonds, may also improve the adsorption capacity of GO towards POPs. The competitive adsorption process revealed that in addition to the direct adsorption onto the GO surface, the molecular aggregation may be another indirect adsorption way existed in the mixed system. Understanding the interaction between GO and POPs in aqueous so-
\end{abstract}

收稿日期: 2019-07-23; 收到修改稿日期：2019-09-23

基金项目: 国家重点研发计划(2017YFA0207002, 2018YFB0504400); 国家自然科学基金(21777039); 中央高校基础研究经 费(2017YQ001); 天津市自然科学基金(青年项目)(16JCQNJC05100)

National Key Research and Development Program of China (2017YFA0207002, 2018YFB0504400); National Natural Science Foundation of China (21777039); Fundamental Research Funds for the Central Universities (2017YQ001); Natural Science Foundation of Tianjin (16JCQNJC05100)

作者简介：赵超锋(1995-), 男, 硕士研究生. E-mail: cfzhao@ncepu.edu.cn ZHAO Chaofeng (1995-), male, Master candidate. E-mail: cfzhao@ncepu.edu.cn

通讯作者: 艾玥洁, 副教授. E-mail: aiyuejie@ncepu.edu.cn AI Yuejie, associate professor. E-mail: aiyuejie@ncepu.edu.cn 
lution is critical to the design and application of graphene-based materials and our findings are believed to contribute further theoretical basis to the engineering treatment of POPs-containing waste water.

Key words: phenolic organic pollutants; graphene oxide; competitive adsorption; molecular dynamics simulation

水资源是地球上一切生命活动赖以生存的自然 资源，随着工业化和城市化的快速发展，工业废水 和生活污水被肆意排放, 大量的酚类有机污染物 (Phenolic Organic Pollutants, POPs)进入自然环境中, 造成严重的水体污染 ${ }^{[1]}$ 。这些 POPs 进入生态系统之 后，能够在动植物体内逐渐富集并通过食物链的传 递最终进入人体, 对人体的消化系统、泌尿系统以 及神经系统产生毒害作用, 从而严重威胁人类的健 康 $^{[2-4]}$ 。因此, 高效去除自然界水体中的 POPs 是当 前亟待解决的环境问题。目前, 对于含 POPs 水体的 处理方法主要有吸附法 ${ }^{[5-7]}$ 、萃取法 ${ }^{[8-9]}$ 、膜分离 法 ${ }^{[10-11]}$ 、生物降解法 ${ }^{[12-13]}$ 以及催化氧化法 ${ }^{[14-15]}$ 等。 吸附法具有操作简单、经济成本低、吸附容量大以 及去除效率高等优势, 在含 POPs 水体的处置中显 示出良好的去除效果, 因而被广泛应用于含 POPs 的污水净化工艺中。而吸附剂又直接影响到吸附法 的应用实效。因此，篮选出吸附容量大、稳定性强、 成本低廉的吸附剂是治理 POPs 污染水体的重要课 题。氧化石墨烯(Graphene Oxide, GO) 是一种二维碳 基材料, 具有较高的比表面积、优异的化学稳定性 以及大量的羟基、羧基等含氧官能团, 并且水溶性 良好, 从而引起了广泛关注 ${ }^{[16-18]}$ 。研究表明 GO 对 POPs 有良好的去除能力 ${ }^{[19-20]}$, 例如, 对苯酚的吸附 量可达 $56.56 \mathrm{mg} / \mathrm{g}^{[21]}$, 对 $\alpha$-䒺酚的吸附量高达 $207.66 \mathrm{mg} / \mathrm{g}^{[22]}$ 。然而, 其内在吸附机理, 尤其当多 种 POPs 共存时, GO 的选择性吸附以及 POPs 之间 的竞争吸附机制尚不明确。

分子动力学模拟(Molecular dynamics simulation, $\mathrm{MD}$ ) 是一种基于经典牛顿力学方程的分子模拟方法,
被广泛应用于药物设计 ${ }^{[23]}$ 、材料 ${ }^{[24]}$ 和生物 ${ }^{[25]}$ 等领 域。在环境领域, MD 模拟可用于研究目标污染物分 子在各种材料界面上的吸附行为 ${ }^{[26-27]}$ 。大量的研究 工作采用 $\mathrm{MD}$ 模拟方法从分子水平上探究吸附过程 中材料与目标污染物的相互作用机制，对不同条件 下的实验结果给予了很好的解释和预测 [5,21,28-29]。由 于吸附过程的复杂性, 已有的实验和理论研究大多 针对单一污染物在材料表面的吸附特性, 而在实际 应用中往往达不到材料预期的实验效果。因此，研 究多种污染物共存时的吸附行为对于材料的实际应 用更有价值。

本工作以苯酚(phenol)、 $\alpha$-蒜酚 ( $\alpha$-naphthol) 和 4辛基酚(4-octyl-phenol)为代表，采用 MD 模拟方法, 研究 POPs 在 GO 表面单独及竞争吸附过程。观察 POPs 在 GO 表面吸附过程中构型的动态变化, 分析 吸附过程中 GO 与 POPs 之间的各种相互作用, 结合 伞形采样的方法计算它们的吸附自由能，从而推断 POPs 在 GO 材料表面吸附的内在作用机理, 为 GO 材料更广泛地应用于含 POPs 水体的治理提供一定 理论依据。

\section{1 计算模型与方法}

研究采用的 GO 模型是二维周期性结构, 根据 Chen 等 ${ }^{[30]}$ 通过实验合成的 $\mathrm{GO}$ 材料 $(\mathrm{O}: \mathrm{C}$ 的比为 $0.12)$, 计算所用的 GO 模型含有 1008 个碳原子和 126 个羟基, $\mathrm{O}$ 与 $\mathrm{C}$ 的原子比为 0.125 。其周期性单元 结构的尺寸为 $5.034 \mathrm{~nm} \times 4.963 \mathrm{~nm}$, 结构如图 1(a)所 示。此外，苯酚、 $\alpha$-䒺酚和 4-辛基酚的结构如图 1(b)
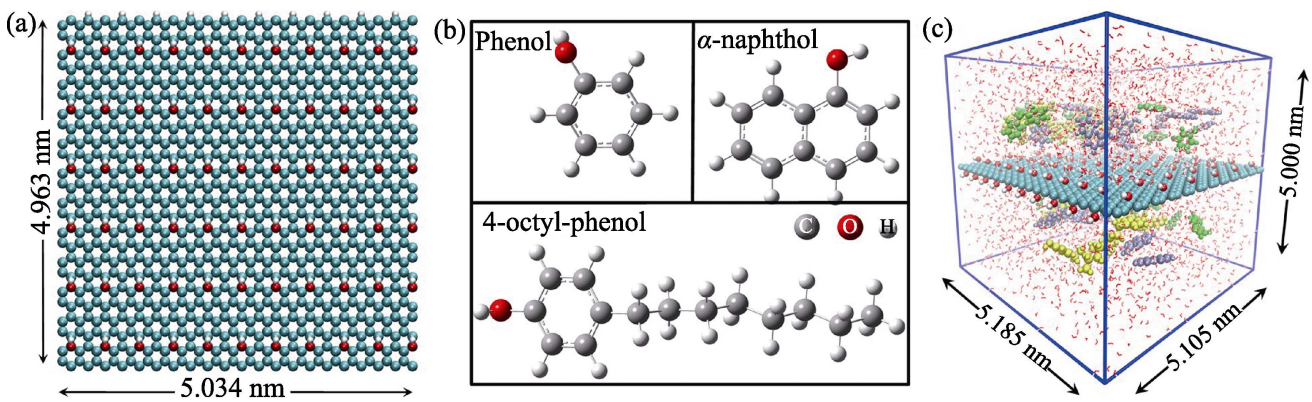

图 $1 \mathrm{MD}$ 模拟采用的(a)GO 模型及其尺寸; (b)苯酚、 $\alpha$-芸酚和 4-辛基酚的结构; (c)立方盒子的尺寸 及苯酚(绿色)、 $\alpha$-䒺酚(紫色)和 4-辛基酚(黄色)在 $\mathrm{GO}$ 表面竞争吸附体系的初始结构

Fig. 1 (a) GO model, (b) structures of phenol, $\alpha$-naphthol and 4-octyl-phenol molecules in MD simulations, and (c) initial configuration of phenol (green), $\alpha$-naphthol (purple) and 4-octyl-phenol (yellow) molecules in the competitive system 
所示。从分子结构的角度来说, 三种分子都具有芳 香环结构和酚着基, 与苯酚相比, $\alpha$-萗酚具有两个 芳香环, 而 4-辛基酚具有较长的柔性烷基链。为了 保证 $\mathrm{GO}$ 结构在 $X$ 和 $Y$ 方向的周期性, MD 模拟采用 $5.158 \mathrm{~nm} \times 5.105 \mathrm{~nm} \times 5.000 \mathrm{~nm}$ 的立方盒子。本研究 采用 Packmol 软件 ${ }^{[31-32]}$ 分别在上述立方盒子中构建 了 $\mathrm{GO}$ 单独吸附苯酚 $(20$ 个)、 $\alpha$-䒬酚( 20 个)和 4 -辛 基酚(20个)体系的初始结构(见图 S1), 以及三种 POPs 竞争吸附体系(每种分子各 20 个)的初始结构 (见图 1(c))。模拟体系采用 $\mathrm{SPC} / \mathrm{E}$ 水分子模型 ${ }^{[33]}$ 。 OPLS-AA 力场是 Jorgensen 等 ${ }^{[34]}$ 开发的一种全原子 力场, 被广泛应用于模拟 $\mathrm{GO}$ 和有机化合物的拓扑 结构 ${ }^{[35-38]}$ 。邢宝山课题组 ${ }^{[21,28-29]}$ 通过 OPLS-AA 力 场成功模拟了多种芳香族有机污染物在 GO 表面的 吸附过程, 并得到了与实验观测相吻合的结果。因 此, 本研究采用 OPLS-AA 力场参数 ${ }^{[34]}$ 构建了 GO 和 三种 POPs 的拓扑结构用于动力学模拟, 计算细节 详见补充材料。所有的分子动力学模拟均采用 GROMACS 5.0.7 软件 ${ }^{[39-40]}$ 完成。

\section{2 结果与讨论}

\section{1 苯酚、 $\alpha$-菜酚和 4-辛基酚在 GO 表面的分布}

经过 $100 \mathrm{~ns}$ 的模拟, 苯酚、 $\alpha$-䒺酚和 4-辛基酚 在 GO 表面单独吸附的体系均达到平衡, 其稳定的 吸附结构如图 2 所示。苯酚和 $\alpha$-菜酚分子具有独特 的平面芳香环结构, 从图 2(a,b)中可以清晰地看到 苯酚和 $\alpha$-菜酚分子平行吸附在 GO 表面。而 4-辛基 酚分子具有较长的烷基链, 从图 2(c)中可以看出它 吸附在 GO 表面的同时还发生分子间聚集, 形成了 团簇。另外, 对于三种 POPs 竞争吸附体系, 从模拟 轨迹中截取了吸附过程中几个典型的吸附结构, 如 图 3 所示。初始结构中三种 POPs 均匀地分布在 GO 两侧。经过 $10 \mathrm{~ns}$ 的模拟, 一部分 4-辛基酚分子直接
吸附在 GO 表面, 其他的 4-辛基酚分子通过与已经 吸附在 GO 表面上的 4-辛基酚分子形成较大的团簇 吸附到 GO 表面。与此同时, 溶液中的苯酚和 $\alpha$-䒺酚 分子也逐渐吸附在 $\mathrm{GO}$ 两侧。吸附到 $25 \mathrm{~ns}$, 溶液中 未能直接吸附到 $\mathrm{GO}$ 两侧的苯酚和 $\alpha$-荎酚分子通过 与 4-辛基酚团簇的相互作用间接地吸附在 GO 表 面。从最终时刻(100 ns)的结构图中可以看出, 溶液 中的苯酚、 $\alpha$-菜酚和 4-辛基酚分子都有效地吸附到 GO 的两侧。由于吸附位点的限制, 使得某些 POPs 吸附时不能够完全平行吸附于 $\mathrm{GO}$ 平面, 而是通过 分子间相互作用形成的团簇构成了间接吸附的外层 吸附层。

从竞争吸附体系中每一个 POPs 分子与 $\mathrm{GO}$ 之 间的质心距离(见图 4)可以发现以下几点特征: (1)吸 附的类型主要有两类, 一类是 POPs 与 $\mathrm{GO}$ 的直接吸 附, 吸附距离在 $0.5 \mathrm{~nm}$ 以内且波动小, 吸附比较稳 定; 另一类是第一吸附层外的间接吸附，吸附距离 在 0.5 2.0 nm 之间且波动大, 稳定性较差。(2)结合 分子动力学的吸附结构以及三种分子的吸附距离可 以看出， $\alpha$-䒺酚和 4-辛基酚相比于苯酚而言, 具有 较强的吸附竞争性和稳定性。此外, 分析竞争体系 中苯酚、 $\alpha$-蒜酚、4-辛基酚两两之间的相互作用能 量(如图 5(a)所示)可以看出, 苯酚和 $\alpha$-䒬酚在与 4辛基酚团簇相互作用的间接吸附过程中存在明显的 竞争关系。 $\alpha$-䒬酚和 4-辛基酚之间的相互作用逐渐 增强, 使得溶液中的 $\alpha$-荎酚能够与 $\mathrm{GO}$ 表面上 4-辛 基酚团簇紧密结合，促进了 $\alpha$-芸酚的吸附。相反，苯 酚与 4-辛基酚之间的相互作用逐渐减弱，在一定程 度上降低了苯酚的吸附效率。

通过分析吸附过程中 POPs 的聚集程度和径向 分布函数, 进一步探究了 POPs 在 GO 表面的分布。 在单独吸附体系中(图 5(b)), 最大的 4-辛基酚团簇 中分子数的平均值为 11.7 , 远大于苯酚(3.6)和 $\alpha$-萗 酚(8.0), 说明 4-辛基酚发生了明显的聚集。而在竞
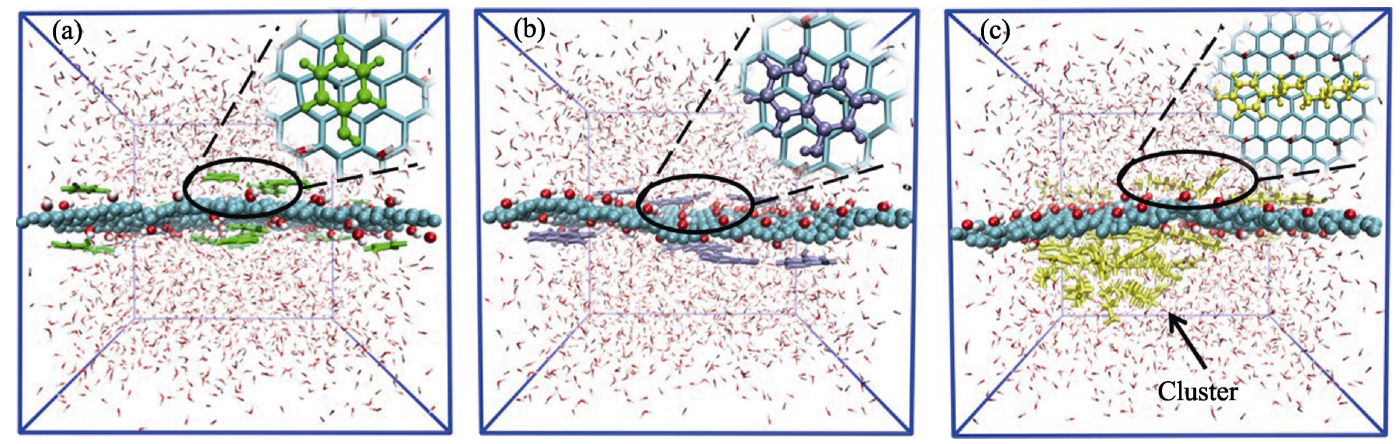

图 2 (a)苯酚、 (b) $\alpha$-菜酚和(c)4-辛基酚在 $\mathrm{GO}$ 表面单独吸附的平衡结构

Fig. 2 Equilibrium structures of (a) phenol, (b) $\alpha$-naphthol and (c) 4-octyl-phenol molecules adsorbed on GO surface 


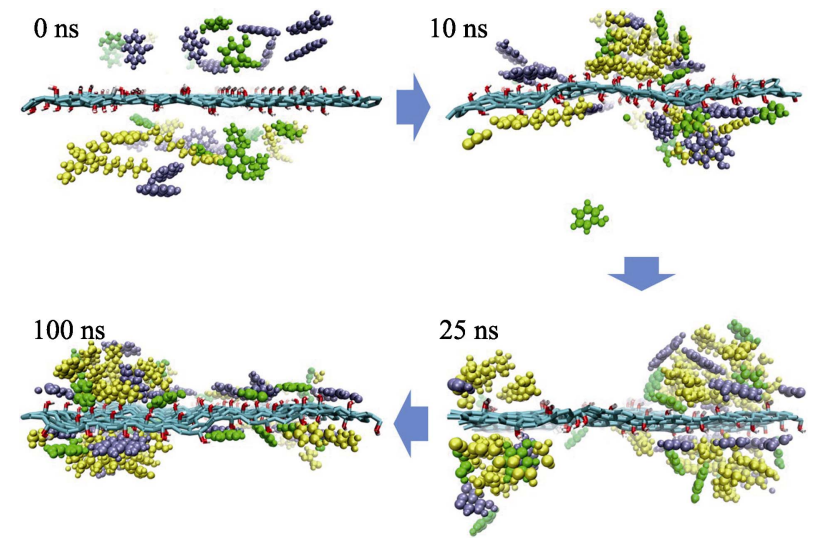

图 3 竞争吸附体系中苯酚(绿色)、 $\alpha$-蒜酚(紫色)和 4-辛基酚 (黄色)在 GO 表面不同时刻的吸附结构图

Fig. 3 Snapshots of competitive system from simulation process at different time

Phenol, $\alpha$-naphthol and 4-octyl-phenol molecules are shown as green, purple and yellow molecules, respectively. Water molecules are not shown to highlight the configuration
争吸附体系中(图 5(c)), 苯酚、 $\alpha$-䒺酚和 4-辛基酚最 大的团簇中分子数的平均值分别为 2.6、4.4 和 9.2, 均比它们单独吸附时的小, 表明多种 POPs 共存时, 不同分子之间的相互影响会降低它们的聚集程度, 从而影响它们的吸附过程。

单独吸附体系中 $\mathrm{GO}$ 与 POPs 的径向分布函数 $(\mathrm{g}(r))$ 及其积分曲线 $(\mathrm{n}(r))$ 如图 5(d) 所示，从图中可以 发现，苯酚、 $\alpha$-䒺酚和 4-辛基酚的 $\mathrm{g}(r)$ 曲线的整体趋 势是一致的，都仅有一个强度明显的峰分布在 0.2 0.5 nm 范围内, 说明三种 POPs 单独吸附时绝大 多数分子都吸附在 $\mathrm{GO}$ 表面的第一吸附层中。此外, 苯酚和 $\alpha$-菜酚体系中 $\mathrm{g}(r)$ 曲线的尖峰比 4-辛基酚体 系中的强度更大而且更尖锐，表明吸附在 GO 表面 的苯酚和 $\alpha$-菜酚分子的有序度比 4-辛基酚分子的 高。从 $\mathrm{n}(r)$ 曲线可以计算出单独吸附体系中 $94.8 \%$ 的苯酚、 $89.4 \%$ 的 $\alpha$-萗酚、 $77.2 \%$ 的 4-辛基酚出现在
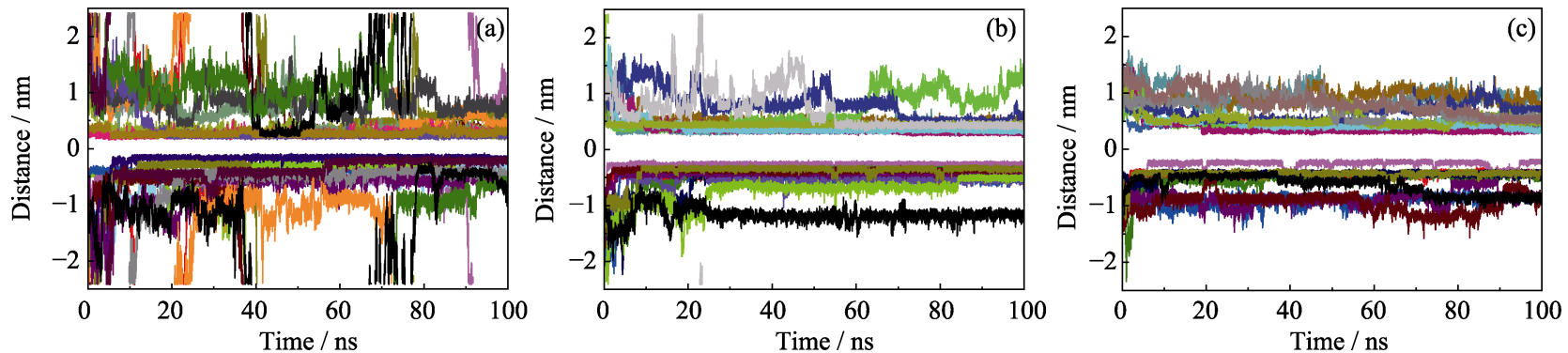

图 4 竞争吸附体系中每一个(a)苯酚、(b) $\alpha$-䒺酚和(c)4-辛基酚分子与 GO 之间质心距离随时间的变化

Fig. 4 Distances of centers of mass between GO and each (a) phenol, (b) $\alpha$-naphthol and (c) 4-octyl-phenol molecule, as function of time
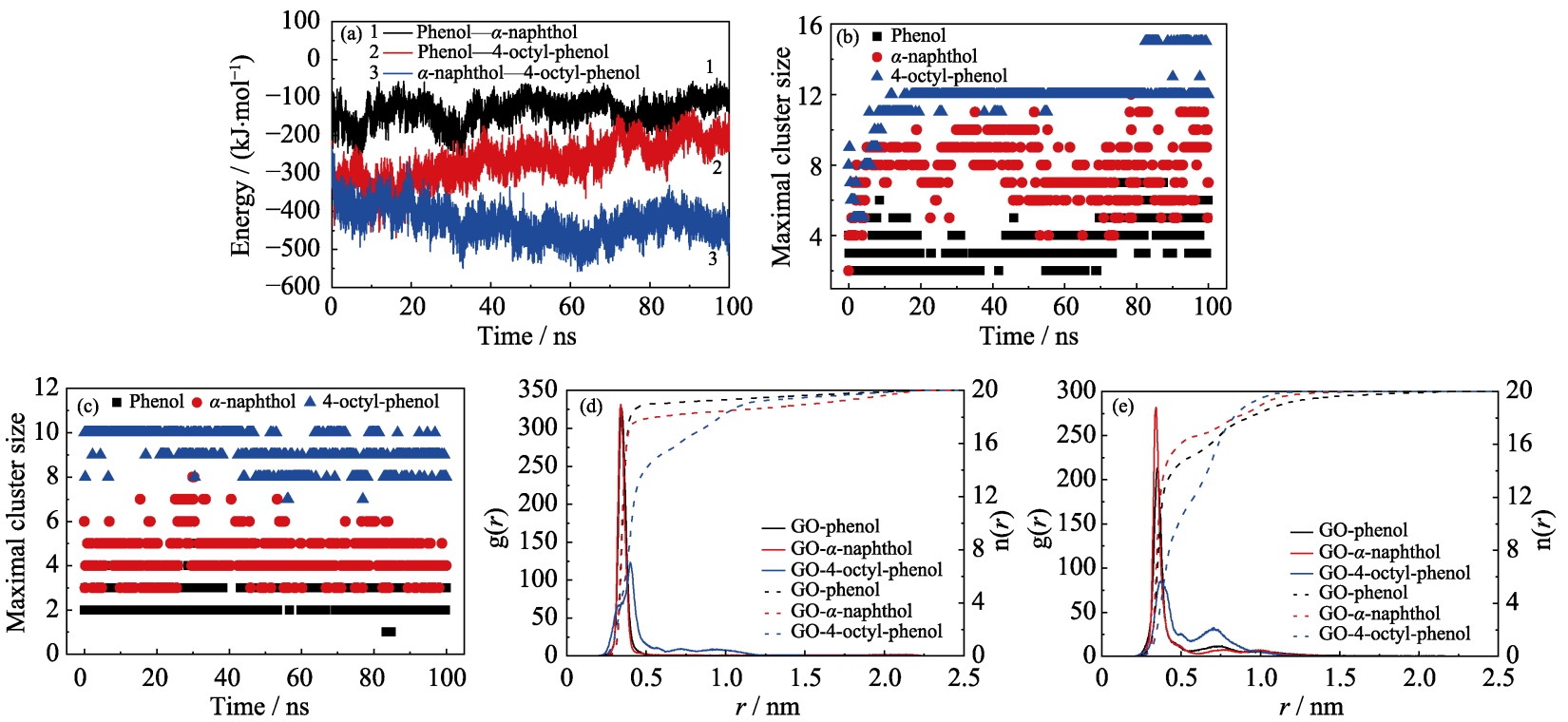

图 5 (a)竞争吸附体系中三种 POPs 之间的相互作用能量, (b)单独和(c)竞争体系中 POPs 最大团簇中的分子数, (d)单独和(e)竞争体系中 POPs 在 GO 表面的径向分布函数曲线 $(\mathrm{g}(r))$ 以及半径 $r$ 范围内相应的分子数目曲线 $(\mathrm{n}(r))$

Fig. 5 (a) Interaction energies between different POPs molecules in competitive system; The maximal cluster size of POPs in

(b) independent and (c) competitive systems, respectively; The radial distribution functions $(\mathrm{g}(r))$ and coordination numbers $(\mathrm{n}(r))$ of POPs in (d) independent and (e) competitive systems, respectively 
$\mathrm{GO}$ 的第一吸附层中, 表明 GO 材料对 POPs 具有较 好的吸附能力, 是一种优良的吸附剂。在竞争吸附 体系中(图 5(e)), 三种 POPs 的 $g(r)$ 曲线中尖峰的强 度比单独吸附体系中明显降低, 说明吸附在 GO 两 侧的 POPs 的有序度明显下降, 这与图 3 的吸附构型 相符合。而且体系中 $74.9 \%$ 的苯酚、 $83.0 \%$ 的 $\alpha$-䒺酚、 $58.3 \%$ 的 4-辛基酚分子出现在 $\mathrm{GO}$ 的第一吸附层中, 与它们单独吸附时的含量相比均明显下降, 说明 GO 表面吸附位点的限制降低了 POPs 的吸附量。此外, $\mathrm{Yu}$ 等 ${ }^{[5]}$ 通过批实验和光谱分析探究了苯酚和 $\alpha$-䒺酚 在还原氧化石墨烯(rGO)表面的竞争吸附行为。他们 的结果表明, 苯酚和 $\alpha$-䒺酚在吸附过程中存在明显 的竞争关系, 最大吸附量比在单独吸附体系中均有 所下降。理论计算研究揭示了 $\alpha$-䒬酚由于具有较强 的疏水性, 在竞争吸附过程中具有较强的优势, 说 明 POPs 分子的疏水性在其竞争吸附过程中占主导。
这与我们计算的 POPs 的疏水性强弱顺序以及吸附 过程主要驱动力的结论相一致。

\subsection{GO 分别与苯酚、 $\alpha$-慕酚、4-辛基酚的相 互作用}

通过伞形采样，如图 6(a)所示，计算了苯酚、 $\alpha$-萗酚和 4-辛基酚在 $\mathrm{GO}$ 表面的吸附能分别为 19.31、33.23 和 $41.34 \mathrm{~kJ} / \mathrm{mol}$ 。吸附能的差异与它们 自身的分子属性有很大的关联。与苯酚相比, 芳香 环的增加 ( $\alpha$-萗酚), 柔性长烷基链(4-辛基酚)等都能 有效地增强分子与 $\mathrm{GO}$ 的相互作用，从而提高吸附 性能。许多实验研究发现, GO 能够有效地去除溶液 中的 POPs 分子 ${ }^{[30,41]}$ 。Zhou 等 ${ }^{[20]}$ 通过改进的 Hummers 方法结合溶剂热法以及两步聚合反应成功 制备了 $\mathrm{Fe}_{3} \mathrm{O}_{4} @$ 聚苯胺修饰的磁性氧化石墨烯材料 $\left(\mathrm{Fe}_{3} \mathrm{O}_{4} @ \mathrm{PANI}-\mathrm{GO}\right)$, 该材料在水溶液中酚类有机污 染物的吸附实验中表现出良好的吸附容量和优异的
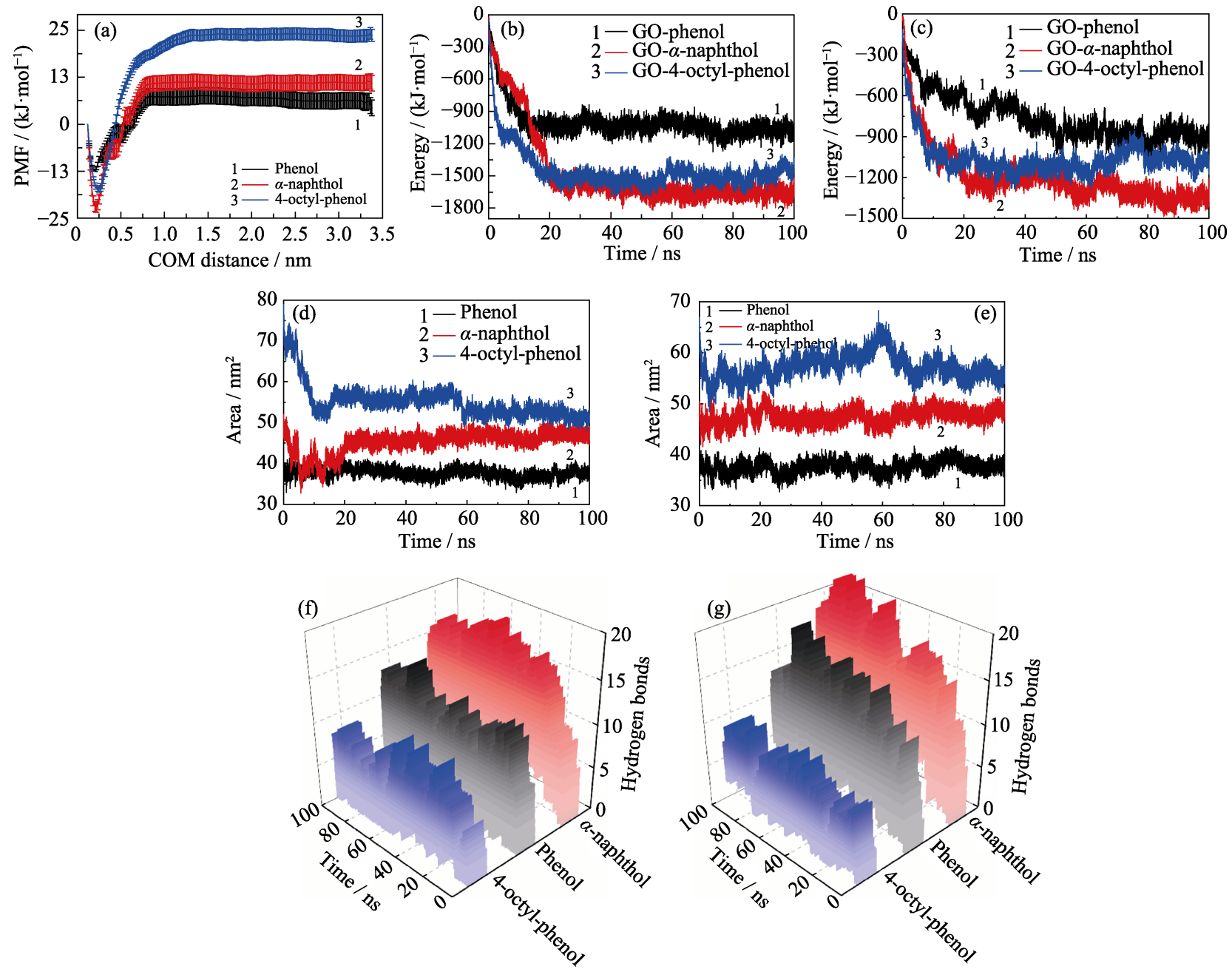

图 6 (a)苯酚、 $\alpha$-菜酚和 4-辛基酚分子的吸附自由能; GO 与 POPs 在(b)单独和(c)竞争体系中的相互作用能量;

POPs 在(d)单独和(e)竞争体系中的疏水面积; GO 与 POPs 官能团在(f)单独和 $(\mathrm{g})$ 竞争体系中形成的氢键数目

Fig. 6 (a) Potential of mean force of POPs molecules; The interaction energies between GO and POPs molecules in (b) independent and (c) competitive systems, respectively; The hydrophobic areas of POPs molecules in (d) independent and (e) competitive systems, respectively; The hydrogen bonds between GO and POPs molecules in (f) independent and (g) competitive systems, respectively 
磁性分离特性。研究结果表明, 该材料对 $\alpha$-䒺酚和 4-辛基酚的最大吸附量分别为 13.19 和 $24.15 \mathrm{mg} / \mathrm{g}$ 。

与 $\alpha$-菜酚相比, 4-辛基酚分子的柔性烷基链能够有 效地增强它与吸附剂材料的相互作用。该实验结果 与我们模拟的吸附过程中 4-辛基酚由于具有较长的 柔性烷基链使其吸附能大于 $\alpha$-菜酚这一理论计算结 果相吻合。

图 6(b,c)分别为单独和竞争吸附体系中 $\mathrm{GO}$ 与 POPs 之间的相互作用能量, 包括库仑相互作用能 (Coulomb interaction) 和兰纳德 - 琼斯势能 (Lennard-Jones Potential, L-J Potential)。其中, 库仑 相互作用能的大小可以直接反映出吸附过程中 $\mathrm{GO}$ 与 POPs 之间静电相互作用的强弱; 而 L-J 势能则是 通过计算粒子间的色散力来体现它们之间范德华相 互作用的大小。从图 6(b)中可以看出单独吸附体系 中 $\mathrm{GO}$ 与三种 POPs 之间的相互作用能量的变化趋 势相似: 在前 $20 \mathrm{~ns}$ 内迅速下降, 随后趋于稳定。最 终, GO 与苯酚、 $\alpha$-䒺酚、4-辛基酚之间的相互作用能 量分别稳定在 $-1073.91 、-1655.69$ 和 $-1492.28 \mathrm{~kJ} / \mathrm{mol}$ (取 80 100 ns 模拟时间范围内相互作用能量的平均 值)。而竞争吸附体系中(见图 6(c)), 在 0 60 ns 内 GO 与苯酚之间的相互作用能量缓慢降低, 最终稳 定在- $891.58 \mathrm{~kJ} / \mathrm{mol} ; \mathrm{GO}$ 与 $\alpha$-萗酚和 4-辛基酚的相互 作用能量均在前 $20 \mathrm{~ns}$ 内迅速降低, 吸附达到平衡 时其相互作用能量分别为 -1355.32 和 $-1075.89 \mathrm{~kJ} / \mathrm{mol}$ 。 将图 6(b,c)中 GO 与 POPs 在不同时刻的库仑相互作 用能、 L-J 势能以及总的相互作用能量分别汇总在 表 $\mathrm{S} 1$ 和表 $\mathrm{S} 2$ 中, 从表中可以清楚地看出单独和竞 争吸附体系中 GO 与 POPs 的 L-J 势能分别约占其总 能量的 $80 \%$ 和 $75 \%$ 以上, 均远大于其库仑相互作用 能, 表明吸附过程中 GO 与 POPs 之间的范德华相互 作用大于它们之间的静电相互作用。仔细比较可以 发现, 竞争吸附体系中 GO 与 POPs 的 L-J 势能明显 比它们单独吸附体系中的小, 而库仑相互作用能却 有所增加, 这说明多种 POPs 共存时, 静电相互作用 在吸附过程中的影响增强了。

单独和竞争吸附过程中 POPs 团簇的溶剂可及 表面积(Solvent Accessible Surface Area, SASA)如图 $\mathrm{S} 2$ 和图 S3 所示。从图中可以看出单独和竞争吸附 过程中三种 POPs 团簇的疏水面积都远大于其亲水 面积。为了更直观地比较疏水作用对 POPs 吸附过 程的影响, 我们将它们在单独和竞争吸附过程中疏 水面积随时间变化的曲线汇总在图 $6(\mathrm{~d}, \mathrm{e})$ 中, 从图 中可以发现, 这三种 POPs 团簇在单独和竞争吸附 体系中疏水作用强弱顺序均为: 4-辛基酚 $>\alpha$-荎酚 $>$ 苯酚。分析可知, 三种分子疏水面积的差异主要来 源于其分子结构本身, 4-辛基酚分子的长烷基链大 大增加了它的疏水面积, $\alpha$-䒺酚结构中苯环数的增
多使其疏水面积大于苯酚。

$\mathrm{GO}$ 表面的羟基官能团能够与 POPs 形成氢键。 图 6(f,g)分别为单独和竞争吸附体系中 $\mathrm{GO}$ 与 POPs 之间形成的氢键数目。在单独(竞争)吸附体系中, $\mathrm{GO}$ 与苯酚、 $\alpha$-蒜酚、4-辛基酚分子在模拟的 $100 \mathrm{~ns}$ 内形 成氢键数目的平均值分别为 $4.9(8.0) 、 9.7(11.2)$ 和 3.7(4.2)。GO 与 POPs 之间形成的氢键在一定程度 上促进了溶液中 POPs 在 $\mathrm{GO}$ 表面上的吸附过程, 而 且在竞争吸附体系中氢键对吸附过程的影响明显 增强。

\section{3 结论}

采用分子动力学模拟的方法, 以苯酚、 $\alpha$-菜酚和 4-辛基酚为代表, 系统研究了 POPs 在 GO 表面的吸 附行为。研究结果表明: (1)在单独吸附的体系中, 溶 液中绝大多数的 POPs 都能够稳定吸附在 GO 表面的 第一吸附层中。(2)POPs 的疏水作用在吸附过程中占 主导, 分子团簇、范德华相互作用、静电相互作用以 及氢键等在一定程度上也能够促进 POPs 在 GO 表面 上的吸附。(3)三种 POPs 共存时存在明显的竞争吸附 现象, 4-辛基酚由于较强的疏水性和较大的吸附能使 其竞争吸附能力最强, $\alpha$-荎酚次之, 苯酚最弱。本研 究为研究环境中 POPs 在 GO 材料上的吸附行为提供 了新的思路和理论基础。

\section{补充材料:}

相关补充材料可以查阅 https://doi.org/10.15541/ jim20190377。

\section{参考文献:}

[1] AHMARUZZAMAN M. Adsorption of phenolic compounds on low-cost adsorbents: a review. Advances in Colloid and Interface Science, 2008, 143(1/2): 48-67.

[2] BABICH H, DAVIS D L. Phenol: a review of environmental and health risks. Regulatory Toxicology and Pharmacology, 1981, 1(1): 90-109.

[3] KARTHIKEYAN K G, CHOROVER JON, BORTIATYNSKI JACKIE M, et al. Interaction of 1-naphthol and its oxidation products with aluminum hydroxide. Environmental Science \& Technology, 1999, 33(22): 4009-4015.

[4] PONZO OSVALDO J, SILVIA CARBONE. Evidence of reproductive disruption associated with neuroendocrine changes induced by UV-B filters, phthalates and nonylphenol during sexual maturation in rats of both gender. Toxicology, 2013, 311(1/2): 41-51.

[5] YU SHU-JUN, WANG XIANG-XUE, YAO WEN, et al. Macroscopic, spectroscopic, and theoretical investigation for the interaction of phenol and naphthol on reduced graphene oxide. Environmental Science \& Technology, 2017, 51(6): 3278-3286.

[6] YU SHU-JUN, WANG XIANG-XUE, AI YUE-JIE, et al. Experimental and theoretical studies on competitive adsorption of aromatic compounds on reduced graphene oxides. Journal of Materials Chemistry A, 2016, 4(15): 5654-5662. 
[7] PAN BO, LIN DAO-HUI, MASHAYEKHI HAMID, et al. Adsorption and hysteresis of bisphenol a and $17 \alpha$-ethinyl estradiol on carbon nanomaterials. Environmental Science \& Technology, 2008, 42(15): 5480-5485.

[8] RUESGAS-RAMON MARIANA, FIGUEROA-ESPINOZA MARIA CRUZ, DURAND ERWANN. Application of deep eutectic solvents (des) for phenolic compounds extraction: overview, challenges, and opportunities. Journal of Agricultural and Food Chemistry, 2017, 65(18): 3591-3601.

[9] CIULU MARCO, CADIZ-GURREA MARIA DE LA LUZ, SEGURA-CARRETERO ANTONIO. Extraction and analysis of phenolic compounds in rice: a review. Molecules, 2018, 23(11): 2890-1-20.

[10] CASTRO-MUNOZ ROBERTO, YANEZ-FERNANDEZ JORGE, FILA VLASTIMIL. Phenolic compounds recovered from agro-food by-products using membrane technologies: an overview. Food Chemistry, 2016, 213: 753-762.

[11] RAZA WASEEM, LEE JECHAN, RAZA NADEEM, et al. Removal of phenolic compounds from industrial waste water based on membrane-based technologies. Journal of Industrial and Engineering Chemistry, 2019, 71: 1-18.

[12] ZYSZKA-HABERECHT BEATA, NIEMCZYK EMILIA, LIPOK JACEK. Metabolic relation of cyanobacteria to aromatic compounds. Applied Microbiology and Biotechnology, 2019, 103(3): $1167-1178$

[13] SINGH PRIYARAGINI, KUMAR RAKESH. Critical review of microbial degradation of aromatic compounds and exploring potential aspects of furfuryl alcohol degradation. Journal of Polymers and the Environment, 2019, 27(5): 901-916.

[14] LUO XU-BIAO, DENG FANG, MIN LU-JUAN, et al. Facile one-step synthesis of inorganic-framework molecularly imprinted $\mathrm{TiO}_{2} / \mathrm{WO}_{3}$ nanocomposite and its molecular recognitive photocatalytic degradation of target contaminant. Environmental Science \& Technology, 2013, 47(13): 7404-7412.

[15] SHAO PENG-HUI, TIAN JIA-YU, YANG FENG, et al. Identification and regulation of active sites on nanodiamonds: establishing a highly efficient catalytic system for oxidation of organic contaminants. Advanced Functional Materials, 2018, 28(13): 1705295.

[16] BORTHAKUR PRIYAKSHREE, BORUAH PURNA K, DAS MANASH R, et al. Adsorption of $17 \alpha$-ethynyl estradiol and $\beta$-estradiol on graphene oxide surface: an experimental and computational study. Journal of Molecular Liquids, 2018, 269: 160-168.

[17] MOLLA ANIRUDDHA, LI YUAN-YUAN, MANDAL BIKASH, et al. Selective adsorption of organic dyes on graphene oxide: theoretical and experimental analysis. Applied Surface Science, 2019, 464: 170-177.

[18] THAKUR KIRTI, KANDASUBRAMANIAN BALASUBRAMANIAN. Graphene and graphene oxide-based composites for removal of organic pollutants: a review. Journal of Chemical \& Engineering Data, 2019, 64(3): 833-867.

[19] MUKHERJEE MALOSHREE, GOSWAMI SUDIPTA, BANERJEE PRIYA, et al. Ultrasonic assisted graphene oxide nanosheet for the removal of phenol containing solution. Environmental Technology \& Innovation, 2019, 13: 398-407.

[20] ZHOU QING-XIANG, WANG YU-QIN, XIAO JUN-PING, et al. Fabrication and characterisation of magnetic graphene oxide incorporated $\mathrm{Fe}_{3} \mathrm{O}_{4} @$ @polyaniline for the removal of bisphenol A, $t$-octyl-phenol, and alpha-naphthol from water. Scientific Reports, 2017, 7(1): 11316.

[21] TANG HUAN, ZHAO YING, SHAN SU-JIE, et al. Theoretical insight into the adsorption of aromatic compounds on graphene oxide. Environmental Science: Nano, 2018, 5(10): 2357-2367.

[22] ZHENG HUI-LING, GAO YANG, ZHU KAI-RUO, et al. Investigation of the adsorption mechanisms of $\mathrm{Pb}$ (II) and 1-naphthol by beta-cyclodextrin modified graphene oxide nanosheets from aqueous solution. Journal of Colloid and Interface Science, 2018, 530: $154-162$.

[23] AMINPOUR MARAL, MONTEMAGNO CARLO, TUSZYNSKI JACK A. An overview of molecular modeling for drug discovery with specific illustrative examples of applications. Molecules, DOI: 10.3390/molecules 24091693

[24] FENG DAI-LI, FENG YAN-HUI, QIU LIN, et al. Review on nanoporous composite phase change materials: fabrication, characterization, enhancement and molecular simulation. Renewable \& Sustainable Energy Reviews, 2019, 109: 578-605.

[25] SPONER JIRI, BUSSI GIOVANNI, KREPL MIROSLAV, et al. RNA structural dynamics as captured by molecular simulations: a comprehensive overview. Chemical Reviews, 2018, 118(8): 4177-4338.

[26] MA ZHAO-YANG, PATHEGAMA GAMAGE RANJITH, RATHNAWEERA THARAKA, et al. Review of application of molecular dynamic simulations in geological high-level radioactive waste disposal. Applied Clay Science, 2019, 168: 436-449.

[27] LIU LU-MENG, LIU JUN-JIE, PEI JING-JING. Towards a better understanding of adsorption of indoor air pollutants in porous mediafrom mechanistic model to molecular simulation. Building Simulation, 2018, 11(5): 997-1010.

[28] TANG HUAN, ZHAO YING, YANG XIAO-NAN, et al. Understanding the $\mathrm{pH}$-dependent adsorption of ionizable compounds on graphene oxide using molecular dynamics simulations. Environmental Science: Nano, 2017, 4(10): 1935-1943.

[29] TANG HUAN, ZHAO YING, SHAN SU-JIE, et al. Wrinkle- and edge-adsorption of aromatic compounds on graphene oxide as revealed by atomic force microscopy, molecular dynamics simulation, and density functional theory. Environmental Science \& Technology, 2018, 52(14): 7689-7697.

[30] CHEN XIAO-XIAO, CHEN BAO-LIANG. Macroscopic and spectroscopic investigations of the adsorption of nitroaromatic compounds on graphene oxide, reduced graphene oxide, and graphene nanosheets. Environmental Science \& Technology, 2015, 49(10): 6181-6189.

[31] MART NEZ JOS MARIO, MART NEZ LEANDRO. Packing optimization for automated generation of complex system's initial configurations for molecular dynamics and docking. Journal of Computational Chemistry, 2003, 24(7): 819-825.

[32] MART NEZ LEANDRO, ANDRADE RICARDO, BIRGIN ERNESTO G, et al. PACKMOL: a package for building initial configurations for molecular dynamics simulations. Journal of Computational Chemistry, 2009, 30(13): 2157-2164.

[33] BERENDSEN H J C, GRIGERA J R, STRAATSMA T P. The missing term in effective pair potentials. Journal of Physical Chemistry, 1987, 91(24): 6269-6271.

[34] JORGENSEN WILLIAM L, MAXWELL DAVID S, TIRADORIVES JULIAN. Development and testing of the OPLS all-atom force field on conformational energetics and properties of organic liquids. Journal of the American Chemical Society, 1996, 118(45): 11225-11236.

[35] WANG JUN-MEI, HOU TING-JUN. Application of molecular dynamics simulations in molecular property prediction I: density and heat of vaporization. Journal of Chemical Theory and Computation, 2011, 7(7): 2151-2165.

[36] SUBASINGHEGE DON VISAL, DAVID ROLF, DU PU, et al. Interfacial water at graphene oxide surface: ordered or disordered? The Journal of Physical Chemistry B, 2019, 123(7): 1636-1649.

[37] PRICE DANIEL J, BROOKS CHARLES L. Detailed considerations for a balanced and broadly applicable force field: a study of substituted benzenes modeled with OPLS-AA. Journal of Computational Chemistry, 2005, 26(14): 1529-1541.

[38] KAMINSKI GEORGE A. Accurate prediction of absolute acidity constants in water with a polarizable force field: substituted phenols, methanol, and imidazole. The Journal of Physical Chemistry $B, 2005$, 109(12): 5884-5890.

[39] BERENDSEN HERMAN J C, VAN DER SPOEL DAVID, VAN DRUNEN RUDI. GROMACS: a message-passing parallel molecular dynamics implementation. Computer Physics Communications, 1995, 91(1/2/3): 43-56.

[40] VAN DER SPOEL DAVID, LINDAHL ERIK, HESS BERK, et al. GROMACS: fast, flexible, and free. Journal of Computational Chemistry, 2005, 26(16): 1701-1718.

[41] WANG JUN, CHEN BAO-LIANG. Adsorption and coadsorption of organic pollutants and a heavy metal by graphene oxide and reduced graphene materials. Chemical Engineering Journal, 2015, 281: 379-388. 


\title{
氧化石墨烯吸附水体中酚类有机污染物的分子动力学模拟
}

\author{
赵超锋 ${ }^{1}$ ，金佳人 ${ }^{1}$ ，霍英忠 ${ }^{1}$ ，孙 陆 $^{2}$, 艾玥洁 ${ }^{1}$
}

(1. 华北电力大学 环境科学与工程学院, 资源环境系统优化教育部重点实验室, 北京 $102206 ; 2$. 南开大学现代 光学研究所, 天津 300350)

对于氧化石墨烯(Graphene Oxide, GO)单独吸 附苯酚的体系, 首先将 $\mathrm{GO}$ 置于盒子中间, 然后在 $\mathrm{GO}$ 上下两侧各放置 10 个苯酚分子, 再用水分子随 机填充体系。同理, 对于 $\mathrm{GO}$ 单独吸附 $\alpha$-菜酚和 4辛基酚的体系, 将同样数目的 $\alpha$-萗酚和 4-辛基酚均 匀分布在 $\mathrm{GO}$ 的上下两侧。而对于竞争吸附体系, 总 共 60 个分子: 苯酚(20个)、 $\alpha$-菜酚(20 个)和 4-辛基 酚(20 个)被平均放置在 $\mathrm{GO}$ 的两侧。在 $\mathrm{MD}$ 模拟正 式开始之前, 先对体系进行能量最小化, 使得体系 的初始结构充分驰豫, 消除体系中不合理的结构。 随后, 在等温等压系综(NPT)下进行 MD 模拟。我们 采用 V-rescale 热浴法 ${ }^{[1]}$ 控制体系在模拟过程中的温度 保持在 $T=298.15 \mathrm{~K}$, 同时采用 Parrinello-Rahman 压浴 法 $^{[2]}$ 控制体系在模拟过程中的恒压条件 $(P=0.1 \mathrm{MPa})$ 。 此外, MD 模拟运用了周期性边界条件以降低边缘效
应的影响, 并且采用 Particle-Mesh Ewald 方法 ${ }^{[3]}$ 计算 长程静电相互作用, 而对于范德华相互作用则采用 截断法来计算(截断半径为 $1.2 \mathrm{~nm}$ )。分子动力学模拟 步长为 $2 \mathrm{fs}$, 模拟时间为 $100 \mathrm{~ns}$, 每 $2 \mathrm{ps}$ 输出一次模 拟的轨迹用于之后的分析。所有的分子动力学模拟均 采用 GROMACS 5.0.7 软件 ${ }^{[4-5]}$ 完成。

苯酚、 $\alpha$-萗酚和 4-辛基酚分子与 $\mathrm{GO}$ 的吸附能 通过一系列伞形采样模拟来计算平均力势(Potential of Mean Force, PMF)而得到。从 NPT 模拟轨迹中吸 附的平衡阶段挑选出一个单分子稳定吸附结构, 首 先将该吸附结构中的 $\mathrm{GO}$ 固定, 沿垂直于 $\mathrm{GO}$ 平面 方向以 $0.01 \mathrm{~nm} \cdot \mathrm{ps}^{-1}$ 的牵引速率将吸附在 $\mathrm{GO}$ 表面上 的单个苯酚、 $\alpha$-䒺酚和 4-辛基酚分子分别拉动到质 心距离为 $3 \mathrm{~nm}$ 左右的位置, 然后从牵引轨迹中每隔 $0.1 \mathrm{~nm}$ 的间距取一个采样窗口。对于每个选择的采

表 S1 在单独吸附体系中 GO 与苯酚、 $\alpha$-萗酚、4-辛基酚分子在不同时刻的相互作用能量

Table S1 Interaction energies between GO and POPs molecules in independent system at different periods

\begin{tabular}{|c|c|c|c|c|c|c|c|c|c|}
\hline \multirow[b]{2}{*}{ Time/ns } & \multicolumn{3}{|c|}{ Phenol $/\left(\mathrm{kJ} \cdot \mathrm{mol}^{-1}\right)$} & \multicolumn{3}{|c|}{$\alpha$-naphthol $/\left(\mathrm{kJ} \cdot \mathrm{mol}^{-1}\right)$} & \multicolumn{3}{|c|}{ 4-octyl-phenol $/\left(\mathrm{kJ} \cdot \mathrm{mol}^{-1}\right)$} \\
\hline & $\begin{array}{l}\text { Coulomb } \\
\text { interaction }\end{array}$ & $\begin{array}{c}\text { L-J } \\
\text { Potential }\end{array}$ & Total & $\begin{array}{l}\text { Coulomb } \\
\text { interaction }\end{array}$ & $\begin{array}{c}\text { L-J } \\
\text { Potential }\end{array}$ & Total & $\begin{array}{l}\text { Coulomb } \\
\text { interaction }\end{array}$ & $\begin{array}{c}\text { L-J } \\
\text { Potential }\end{array}$ & Total \\
\hline 20 & -44.10 & -866.02 & -910.12 & -317.12 & -1172.76 & -1489.87 & -195.98 & -1311.17 & -1507.15 \\
\hline 40 & -174.71 & -877.44 & -1052.16 & -388.46 & -1276.89 & -1665.35 & -162.60 & -1359.01 & -1521.60 \\
\hline 60 & -124.19 & -897.25 & -1021.45 & -405.37 & -1243.64 & -1649.01 & -96.07 & -1340.12 & -1436.19 \\
\hline 80 & -167.61 & -864.44 & -1032.05 & -358.80 & -1313.62 & -1672.42 & -125.83 & -1454.64 & -1580.47 \\
\hline 100 & -237.23 & -880.63 & -1117.87 & -251.36 & -1338.97 & -1590.33 & -62.78 & -1344.93 & -1407.71 \\
\hline
\end{tabular}

表 S2 在竞争吸附过程中 GO 与苯酚、 $\alpha$-萗酚、4-辛基酚分子在不同时刻的相互作用能量

Table S2 Interaction energies between GO and POPs molecules in competitive system at different periods

\begin{tabular}{|c|c|c|c|c|c|c|c|c|c|}
\hline \multirow[b]{2}{*}{ Time/ns } & \multicolumn{3}{|c|}{ Phenol/(kJ.mol $\left.{ }^{-1}\right)$} & \multicolumn{3}{|c|}{$\alpha$-naphthol $/\left(\mathrm{kJ} \cdot \mathrm{mol}^{-1}\right)$} & \multicolumn{3}{|c|}{ 4-octyl-phenol $/\left(\mathrm{kJ} \cdot \mathrm{mol}^{-1}\right)$} \\
\hline & $\begin{array}{l}\text { Coulomb } \\
\text { interaction }\end{array}$ & $\begin{array}{c}\text { L-J } \\
\text { Potential }\end{array}$ & Total & $\begin{array}{l}\text { Coulomb } \\
\text { interaction }\end{array}$ & $\begin{array}{c}\text { L-J } \\
\text { Potential }\end{array}$ & Total & $\begin{array}{c}\text { Coulomb } \\
\text { interaction }\end{array}$ & $\begin{array}{c}\text { L-J } \\
\text { Potential }\end{array}$ & Total \\
\hline 20 & -160.11 & -448.40 & -608.51 & -235.85 & -914.54 & -1150.38 & -71.34 & -980.56 & -1051.90 \\
\hline 40 & -240.86 & -533.62 & -774.48 & -288.30 & -960.43 & -1248.73 & -182.74 & -928.42 & -1111.16 \\
\hline 60 & -334.12 & -573.27 & -907.39 & -354.11 & -993.34 & -1347.46 & -152.72 & -1013.83 & -1166.55 \\
\hline 80 & -215.40 & -672.38 & -887.78 & -363.20 & -959.82 & -1323.02 & -201.10 & -912.11 & -1113.20 \\
\hline 100 & -214.74 & -674.05 & -888.79 & -305.51 & -1069.33 & -1374.84 & -179.30 & -918.63 & -1097.92 \\
\hline
\end{tabular}



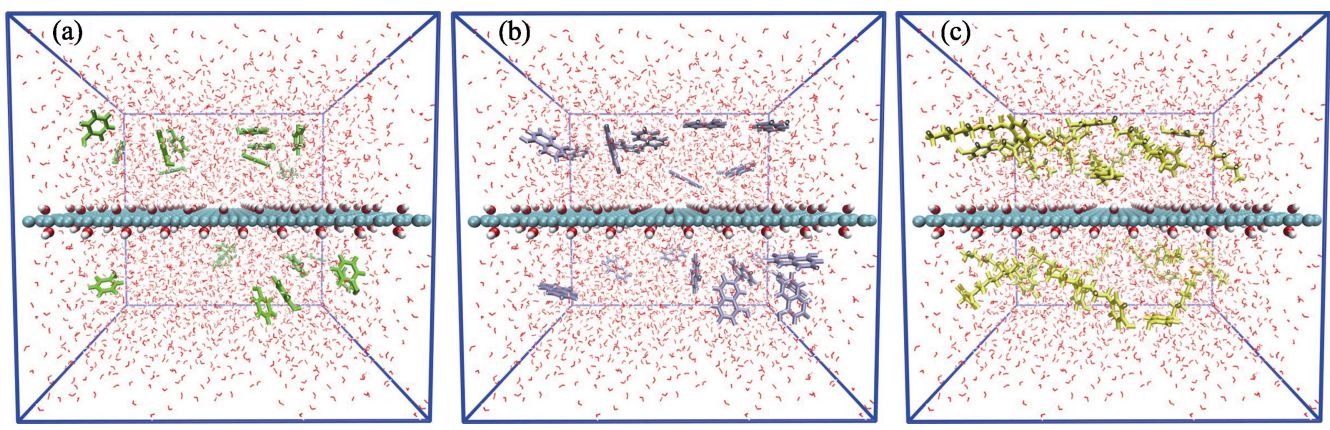

图 S1 (a)苯酚、(b) $\alpha$-菜酚和(c)4-辛基酚单独吸附体系的初始结构图

Fig. S1 Initial configurations of (a) phenol, (b) $\alpha$-naphthol and (c) 4-octyl-phenol molecules in the independent system
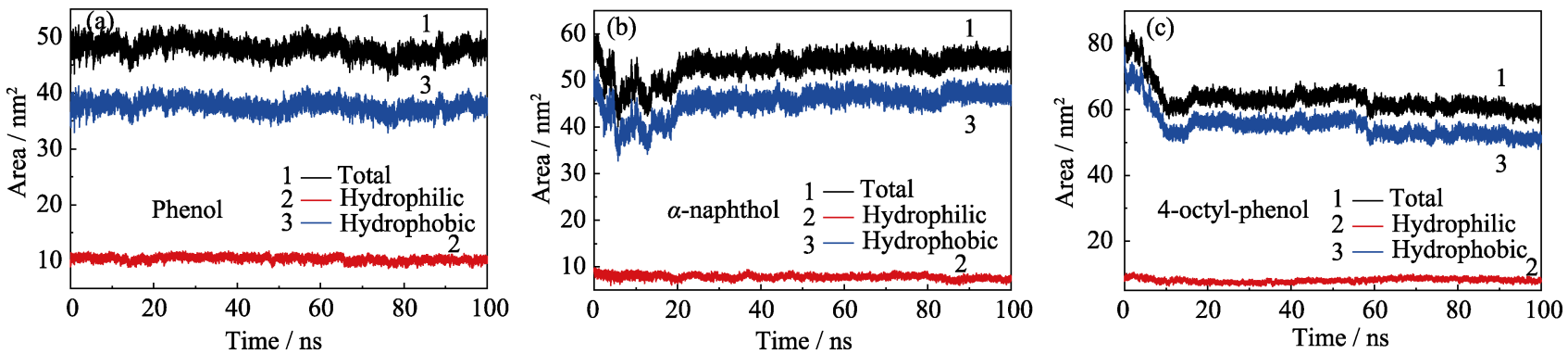

图 S2 单独吸附体系中(a)苯酚、(b) $\alpha$-荎酚和(c)4-辛基酚的溶剂可及表面积

(solvent accessible surface area, SASA) 随时间的变化

Fig. S2 SASAs of (a) phenol, (b) $\alpha$-naphthol and (c) 4-octyl-phenol molecules in the independent system
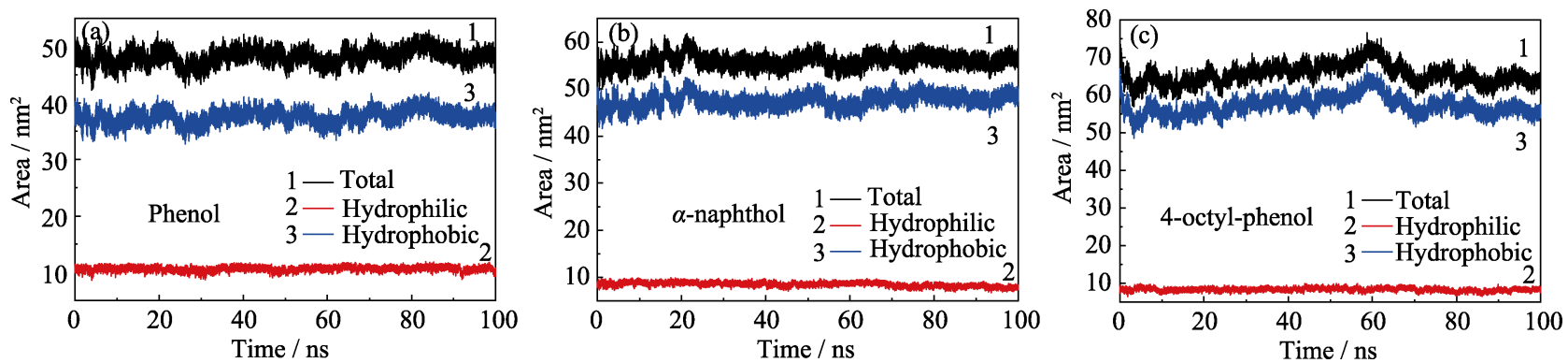

图 S3 竞争吸附体系中(a)苯酚、(b) $\alpha$-萗酚和(c)4-辛基酚的 SASAs 随时间的变化

Fig. S3 SASAs of (a) phenol, (b) $\alpha$-naphthol and (c) 4-octyl-phenol molecules in the competitive system

样窗口, 以弹性系数为 $1000 \mathrm{~kJ} \cdot \mathrm{mol}^{-1} \cdot \mathrm{nm}^{-2}$ 的简谐力 将酚类有机分子限制在选定的窗口中，在 NPT 系综 下进行 $200 \mathrm{ps}$ 的平衡模拟, 再续跑 $10 \mathrm{~ns}$ 用于数据 分析。伞形采样模拟的其他计算细节与 MD 模拟中 使用的参数相同。最后, 采用加权直方图分析方法 (Weighted Histogram Analysis Method, WHAM) ${ }^{[6]}$ 得 到 PMF 并计算吸附能。

\section{参考文献:}

[1] BUSSI GIOVANNI, DONADIO DAVIDE, PARRINELLO MICHELE. Canonical sampling through velocity rescaling. Journal of Chemical Physics, 2007, 126(1): 014101.

[2] PARRINELLO M, RAHMAN A. Polymorphic transitions in single crystals: a new molecular dynamics method. Journal of Applied Physics, 1981, 52(12): 7182-7190.

[3] DARDEN TOM, YORK DARRIN, PEDERSEN LEE. Particle mesh Ewald: an $N \cdot \log (N)$ method for Ewald sums in large systems. Journal of Chemical Physics, 1993, 98(12): 10089-10092.

[4] BERENDSEN HERMAN J C, VAN DER SPOEL DAVID, VAN DRUNEN RUDI. GROMACS: a message-passing parallel molecular dynamics implementation. Computer Physics Communications, 1995, 91(1/2/3): 43-56.

[5] VAN DER SPOEL DAVID, LINDAHL ERIK, HESS BERK, et al. GROMACS: fast, flexible, and free. Journal of Computational Chemistry, 2005, 26(16): 1701-1718.

[6] SOUAILLE MARC, ROUX BENOîT. Extension to the weighted histogram analysis method: combining umbrella sampling with free energy calculations. Computer Physics Communications, 2001, 135(1): 40-57. 